\title{
ATENCION PSICOLÓGICA A LA MUJER MALTRATADA: Una propuesta
}

\author{
*Mg. Rosa Elena Huerta Rosales.
}

Se ilustra un Modelo de Atención Psicológica a la mujer maltratada. Modelo diseñado y practicado por más de doce años en un Centro Materno Infantil del Ministerio de Salud, de la ciudad de Lima. Se sustenta en la psicoterapia gestalt desarrollada por Perls y toma los aportes de la Psicología de la Salud y la Salud Comunitaria que plantean la necesidad de realizar atención primaria (OMS, 1978). Desde un trabajo en equipo multidisciplinario, interinstitucional y en redes hace prioritario internalizar la necesidad de coordinar y trabajar en equipo con la participación activa de la comunidad por lo que es básico construir un marco conceptual integrativo capacitando a Promotoras para la prevención de la Violencia intrafarnjliar a partir de los grupos organizados. De igual forma considera que la intervención debe considerar tanto a la «víctima» como al «agresor». En cuanto a la intervención terapéutica, esta puede ser individual o grupal, y a través de pares.

PALABRAS CLAVES: Violencia Familiar, mujer maltratada, proceso terapéutico, psicoterapia Gestalt

A Model of Psychological Attention to the battered woman acquires knowledge. Model designed and practiced by more than twelve years in an Infantile Maternal Center of the Ministry of Health, the city of Lima. Gestalt is sustained in the psycotherapy developed by Perls and takes the contributions from the Psychology of the Health and the Communitarian Health that raise the necessity to make primary attention (the WHO, 1978). From a work in multidisciplinary equipment, interinstitutional and in networks it makes high-priority internalizar the necessity to coordinate and to work in equipment with the active participation of the community reason why he is basic to construct a integrativo conceptual frame enabling to Promoters for the prevention of the intrafamiliar Violence from the organized groups. Similarly it considers that the intervention must consider so much to the «victim» Iike the «aggressor». As far as the therapeutic intervention, this can be individual or group, and through pairs.

KEY WORDS: Familiar violence, battered woman, therapeutic process, psycotherapy gestalt. 


\section{INTRODUCCIÓN}

Una de las actividades más frecuentes, que enfrentan los profesionales de la salud, es atender los problemas de violencia doméstica, ya sea de manera directa o encubierta. Cabe entonces preguntarse, ¿a que se debe que se tolere el maltrato de manera permanente?, ¿porque ciertos hombres descargan sistemáticamente la agresión contra su cónyuge o su pareja? Tales cuestionamientos se derivan de que el problema de la violencia doméstica en el ámbito familiar ha sido dejado de lado o encubierto durante mucho tiempo por los investigadores de la conducta y la sociedad en general. Las dimensiones y magnitud que alcanza este problema en nuestro país se han invisibilizado probablemente porque se suponía que la familia era y debería ser un medio cálido, afectivo y tranquilo.

En el Perú la violencia doméstica hasta hace poco era aceptada solo para sectores populares. Sin embargo es tan frecuente que afecta a miles de familias, mujeres, niños y ancianos sin diferenciar raza, cultura, religión, ni recursos económicos. Explicitar las dimensiones resulta muy difícil, pues las víctimas, no presentan denuncias ya que se muestran renuentes o avergonzadas a revelar su situación, como si fueran responsables del maltrato que reciben, preocupándose muchas veces en mantenerlo en secreto que hallar una solución. Estos malos tratos infligidos hacia la mujer implican, además, problemas de salud, interrumpen el desarrollo integral de los implicados, siendo los niños los más perjudicados.

La conceptualización de violencia familiar, se refiere a las formas de agresión y abuso que se dan en las relaciones al interior de las familias, de los padres hacia el niño y de la mujer en su relación de pareja. (Son las mujeres y los niños las víctimas más comunes de este abuso). Definición que incluye, también el maltrato hacia el anciano y el hombre.

Es decir que la familia como proveedora de modelos y dc aprendizaje social debe ocupar un lugar preferencial en cualquier estudio o intervención en la violencia intrafamiliar; lo que conlleva a desarrollar y manejar los mismos conceptos y criterios para su intervención.

Por ser la violencia familiar un problema de salud pública que no respeta ni el nivel educativo ni económico de la víctima, nos plantea por un lado, la necesidad de atender de manera eficaz y oportuna y por otro lado la necesidad de trabajar en redes. Si bien es cierto, los sectores gubernamentales y no gubernamentales se vienen esforzando en delinear políticas y estrategias de atención, con avances especialmente en el aspecto legal, aún faltan aunar esfuerzos para atender la demanda, que se ha venido incrementando aunque no como se esperaba, y llegar a todos los lugares del país. Este avance, si cabe el término, lo observamos cuando llegan a los establecimientos de salud niños traídos por la maestra con evidencias de ser maltratado y mujeres que manifiestan que la charla escuchada o la difusión en la radio sobre su «problema» las ha impulsado a solicitar atención.

Por consiguiente, se hace necesario hacer algunas precisiones en lo concerniente a la violencia doméstica, la cual incluye a todas las agresiones físicas, psicológicas y sexuales que sufren las mujeres dentro de la familia, ejercida por los esposos, compañeros, hermanos e hijos. Este tipo de violencia es la más encubierta dentro de la sociedad por los patrones culturales e ideológicos que se manejan, manifestándose a través de:

- Carga excesiva de los quehaceres de la casa. Es la responsable de la conducción de la casa y de los quehaceres (lavado, atención a los hijos, etc.). Estas actividades se consideran como algo innato que forman parte de la condición de mujer.

- La mujer es considerada tanto por los hijos como por el esposo como autoridad de segunda instancia. Aunque la mujer aporte económicamente al hogar. 
- El hombre cree que el haberse casado le da licencia para pegar y ser dueño de ella.

Otros estudios consideran a la situación de marginación, pobreza, hambre, falta de oportunidades y la frustración diaria como las razones que impiden que las relaciones entre los miembros sean saludables.

La familia cumple así, la función de perpetuar la violencia contra la mujer, es la productora de modelos relacionados, en ella se reproducen conductas futuras, se aprenden roles sociales propios de cada sexo. Una familia diseñada con esta forma de pensar y sentir, va determinar roles y funciones diferenciados con respecto a las conductas de varón o mujer.

\section{CARACTERÍSTICAS DE LA FAMILIA QUE VIOLENTAN A LA MUJER}

La mujer victima de la violencia doméstica es miembro de una familia con una determinada organización que a su vez pertenece a un sistema social con una cultura en el cual coexisten mitos, prejuicios, ,valores sobre la estructura familiar y la relación entre sus miembros.

La familia en donde se ejerce la violencia contra la mujer presenta formas jerárquicas de organización, con funciones rígidamente definidas que colocan a la mujer en una situación de subordinada. Estas familias disfuncionales se caracterizan por su rigidez y resistencia al cambio y en ellas la violencia es una condición para mantener este tipo de estructura de dominación.

La violencia que tiene lugar en la familia y en la sociedad, es parte de un mismo sistema. Así, en la medida en que la relación de poder se establece en la familia, el uso de la violencia se condiciona a los individuos como método legítimo para imponer la autoridad entre estos y otros estamentos sociales. Paralelamente, la legitimidad de pautas violentas. en la sociedad avala la presencia de este tipo de interacciones en la familia.

Por otro lado. estudios sobre el estrés familiar (Cohen y Lazarus, 1982; Meichenbaum y Turk, 1984) describen a las tensiones creadas por las perturbaciones del entorno y/o internas de la familia que amenazan el equilibrio familiar y a veces la existencia misma de esta, generando y/o incrementando comportamientos violentos y negligentes.

\section{¿QUE HACE QUE LA MUJER MANTENGA LA SITUACION DE VIOLENCIA?}

Según Long y McNamara (1988) existen tres explicaciones alternativas del porqué una mujer maltratada permanece en la relación de maltrato:

1. El incidente de maltrato puede estar operando como estimulo discriminativo para el reforzamiento positivo; es así que la Dra. Leonore Walker (1979) encontró que la gran parte de las mujeres en su estudio afirmaban que después de un incidente de maltrato, el agresor se mostraba arrepentido y complaciente.

2. El castigo puede aumentar en los sujetos la necesidad de búsqueda de amor y de afecto; desgraciadamente por el aislamiento en el que la mujer se suele encontrar, es el agresor quien satisface esta necesidad.

3. Otro efecto paradójico del castigo es su alta resistencia a la extinción.

Esta alternativa sugiere que soporta el maltrato porque está unido a un fuerte reforzador, el cual tiene mucho valor para ella.

Otras razones que se encuentran son:

- Aprendizaje de victimización, las investigaciones nos señalan que ellas proviene de familias generacionalmente maltratantes.

Síndrome de Estocolmo; La Rouche (1993) refiere que aparece en la víctima un sentimiento positivo hacia su agresor, ello se explica por la dependencia que se da 
entre el agresor y la víctima. La mujer violentada siente aprecio por el agresor que le permite vivir, tiene reconocimiento por no matarla. La víctima siente que el agresor tiene el poder de vida y muerte sobre ella. Aumentando así sus sentimientos de dependencia y pasividad. El agresor contribuye a desarrollar un síndrome por la forma que hace alternar el castigo y el «amor»

El ciclo de la violencia, estudiado por Leonere Walker (1979) quién plantea la existencia de tres etapas a saber; acumulación de tensión, descarga aguda de las tensiones y luna de miel, donde la mujer se queda entrampada.

\section{MITOS Y REALIDAD SOBRE LA VIOLENCIA DOMESTICA}

La cosmovisión familiar está programada intrínsecamente desde la infancia y explica porque sus miembros tienden a repetirla no con el fin de continuarlo sino con el afán de darle solución. (Boscolo y Cecchin, 1982). De esta manera tratan de darle solución a un problema generacional que no logran resolver. La familia puede disminuir o reforzar la interacción agresiva, sin embargo el mito a través de los interacciones de sus miembros va a anular la posibilidad del uso de conductas alternativas a lca violencia, produciéndose interacciones recursivas que se autopereptuan y se refuerzan.

En nuestro medio muchos comportamientos y actitudes están cargados de prejuicios y mitos que conducen a mostrar un comportamiento negativo que daña la salud mental no solo de la victima sino también del agresor.

En el caso del agresor se encuentra que éste también a estado expuesto situaciones de violencia en su familia de origen, presentan además baja autoestima y déficit en habilidades verbales, falta de asertividad, especialmente en relación con sus parejas, y una gran necesidad de ejercer control. Por consiguiente es de necesidad plantear intervención terapéutica no sólo para la víctima sino también para el agresor o maltratan te.

A través de esta descripción se pretende esclarecer una serie de creencias sociales que lamentablemente no solo se encuentran dentro de la población de mujeres maltratadas sino también dentro del grupo de profesionales de salud mental, ya que pueden llegar a confundir las reacciones de victimización con rasgos de personalidad. Este tipo de creencias puede afectar no sólo al paciente sino también a los profesionales en la práctica diana.

En cuanto a los datos estadísticos, por ejemplo en Estados Unidos muestran que el índice de incidencia anual de malos tratos oscila entre un $28 \%$ a un $55 \%$ de la población general (Strauss, 1978). En Canadá en 1986, un tercio de todas las parejas en proceso de separación alegaron como motivo el maltrato físico o psíquico (Lupri, 1989). En nuestro país resulta problemático llegar a conocer el verdadero número de casos de violencia doméstica, los datos que se conocen provienen de instituciones como PROMUDEH, Comisaría de Mujeres y DEMUS, entre alguna de ellas, correspondiendo la mayoría de ellos a los estratos populares y muy pocos a otros sectores. Las cuales al incrementarse las denuncias se incrementan los reportes, es decir que sólo tenemos datos de denuncias.

\section{INTERVENCION TERAPEUTICA}

La propuesta que se propone a continuación toma los aportes de la Psicología de la Salud y la Salud Comunitaria que plantean la necesidad de brindar atención primaria con el fin de proteger y promover la salud de todos los pueblos del mundo (OMS, 1978). Se define a la salud como parte de la vida diaria y dimensión esencial de la calidad de nuestras vidas, dando a las personas la posibilidad de manejar su propia salud, subrayando, además, el papel del individuo y de la comunidad en la definición de su propia salud. Desde esta perspectiva la salud ya no es medida solamente en términos de enfermedad y muerte, por el contrario pasa a ser un estado que tanto individuos como comunidades intentan lograr, 
mantener o recuperar y no como algo que solo sucede como consecuencia de un tratamiento o curación de enfermedades o lesiones. Este aporte conlleva a la necesidad de organizar y coordinar todas nuestras instancias gubernamentales y no gubernamentales para incluir a la salud mental, menoscabada y/o deteriorada por la violencia en todos los aspectos como objetivo primordial y al mismo tiempo partir, desde un trabajo en equipo multidisciplinario, a un trabajo en redes que nos permitirá ser eficaces y oportunos. Por consiguiente la prevención y la promoción de la salud van de la mano con la intervención frente a la problemática de la violencia intrafamiliar que demanda de manera urgente nuestra atención dado ser un serio problema de salud pública.

Asimismo, es menester señalar que no existen profesionales de exclusividad en la atención de la problemática de la violencia intrafamiliar, pues su intervención requiere del conjunto de los profesionales y sectores. Lo primordial es empezar a ser un real integrante de un equipo, tal que, al conocer sus funciones propicie sinergia. Por consiguiente se hace prioritario internalizar la necesidad de coordinar y trabajar en equipo con la participación activa de la comunidad, así como «hablar» un mismo lenguaje.

Para poder intervenir terapéuticamente en los casos de violencia intrafamiliar, a decir de Bronfenbrenner (1987), es necesario no aislar a la persona en estudio de sus entornas naturales (microsistema, exosistema, microsistema, nivel individual) siendo importante construir un marco conceptual integrativo; así como focalizar nuestra intervención tanto en la «víctima» como en el «agresor», pues una política diferente puede conllevar a que no estamos comprendiendo ni aceptando que la mujer desee continuar o ayudar a su pareja y lo que es más grave ver al agresor como un «monstruo» inhumano. Por consiguiente el abordaje terapéutico incluye a la mujer maltratada «víctima»), al agresor ("maltratante») y a la familia.

En cuanto a la intervención terapéutica, esta puede ser individual o grupal, en algunos casos es conveniente trabajar tanto de manera individual como de manera grupal. Simultáneamente con Psicoterapia Individual se pretende que las mujeres víctimas de maltrato puedan manejar mejor el trauma, lograr una mayor sensación de control sobre su cuerpo, mejorar su autoestima, etc.; la Psicoterapia de Grupo permite que el sujeto, al estar en un «microcosmo» pueda aplicarlo al macrocosmo en el que vive, siendo esta modalidad un mejor sistema de apoyo, ya que permite compartir el trauma, establecer relaciones con otros miembros, aleja los sentimientos de desesperanza y vergüenza, aumenta el sentimiento de autoeficacia.

\section{PROPUESTA DE INTERVENCION TERAPEUTICA A LA MUJER MALTRATADA}

\section{Proceso terapéutico en la mujer maltratada (r.e. Huerta, 1998)}

La presente propuesta de Atención Psicológica a la mujer maltratada, es un Modelo de Intervención que se sustenta en la psicoterapia Gestalt desarrollada por Perls; modelo diseñado y practicado por más de doce años en un Centro Materno Infantil del Ministerio de Salud, de la ciudad de Lima; asimismo toma los aportes de la psicología d la salud y la salud comunitaria.

Estos dos relatos nos permiten visualizar la relevancia y pertinencia del modelo a presentar: 
Angélica (37 años), profesora:

.. . hace 16 años mi esposo me maltrata física y psicológicamente ... porque yo era tímida, yo me quedaba callada ... después que me insultaba se iba y me dejaba sola con mis hijos, he intentado matarme, siento que la vida no vale la pena. Me siento desengañada y frustrada

Yolanda (42 años):

... hace 10 años que vengo recibiendo maltrato físico, insultos, humillaciones,... me han tenido que llevar de emergencia al hospital... siento que yo soy la culpable,... tengo miedo, cuando dan las 5 de la tarde empiezo a temblar, grito a mis hijos ...

La atención psicológica parte de la entrevista inicial, del primer contacto que establecemos con ella, es importante apoyar y acompañar la decisión de venir a hablar. Consta de dos etapas:

\section{Primera Etapa, de acogida:}

- Escucha no directiva, para que pueda apoyarse, descargarse, sentirse acogida, hablar y confiar.

- Buscar factores de riesgo a través de indicadores directos (moretones, etc.) e indirectos (labilidad emocional, etc.);. Cuidar en todo momento cuando interrumpir y cómo interrogar, buscando que consultante sea lo más clara y amplia en su relato.

- Es el momento de mayor duración

- Rol del Terapeuta: No efectúa intervenciones, escucha, hace uso del «como SI», para conocer la situación

- Observar la presencia del ciclo de la violencia, propiciando el darse cuenta, aquí y ahora, si es posible.

Dar psicoterapia de apoyo considerando la comunicación analógica (que dice su cuerpo, sus manos su expresión, el tono de su voz, etc., que ella no lo dice). Se hace conveniente en este momento el uso de ejercicios de fantasías dirigidas para descargar culpa, cólera, miedo, etc.

- Establecer la alianza terapéutica, descubriendo, revisando y analizando junto con consultante el tipo de familia en el que ha estado inmersa

- Factores protectores

- Factores de riesgo

\section{Segunda etapa:}

\section{De intervención propiamente dicha:}

- Contrato terapéutico, teniendo en cuenta la presencia o no de problemas psicopatológicos.

- Se determina con mujer el grado de victimización

- Importante plantear las estrategias a seguir para que mujer visualice la necesidad de parar la situación de maltrato.

Actitud Directiva, para confrontar (uso de preguntas en reflejo) a mujer sobre:

- Cómo está viviendo dentro del ciclo de violencia: como se «entrampa»

- Actitud y comportamiento real del esposo o pareja, no la fantaseada.

- Repercusión en sus hijos y consecuencias actuales.

- Incongruencias entre su pensamiento, sus sensaciones y su comportamiento Reforzar defensas 
Se deben incluir preguntas tales como:

- Antecedentes de violencia en familia de origen de la mujer y su pareja.

- Sexualidad de entrevistada y pareja. Primer incidente y peor incidente Denuncias, hasta donde llegaron Parejas anteriores

Habilitación de Competencias:

- Fortalecer autoestima de mujer.

- Implementar habilidades sociales.

- Conectarse con grupos organizados de comunidad

- Seguimiento

- Psicoterapia de grupo

- Capacitarla como Promotora, si reúne el perfil para ello

- Educación a pares en su comunidad, una vez concluida la capacitación y la práctica como Promotora.

Ello implica una atención permanente de parte del terapeuta, escucha y soporte, considerando las creencias, el conjunto de miedos que tienen las mujeres golpeadas y el contexto social dentro del cual se da la entrevista. Para lo cual se hace necesario particularizar los ámbitos y sujetos de competencia que necesitará la mujer maltratada en su tratamiento. Paralelamente en algunos casos y otras posterior a la intervención terapéutica se educa a la mujer para trabajar con sus pares en la comunidad.

Los resultados del presente trabajo son más cualitativos que cuantitativos así observamos mayor demanda de atención en la consulta, el establecimiento de salud ha sido considerado centro de Referencia para la atención de casos de violencia familiar y un gran logro es que la mujer se ha dado cuenta de que su problema puede atenderse y llegar a una solución saludable para ella, su familia y comunidad. Vivos ejemplos de ello son las mujeres de los Asentamientos Humanos que vienen a que les ayuden a «mejorar su autoestima»y las Promotoras al recibir el diploma referir sentir «he crecido, soy importante».

Finalmente, el presente trabajo pretende motivar a los investigadores a estudiar sobre esta problemática álgida y demandante de estrategias de atención en todos los ámbitos; si bien es cierto se ha avanzado en esta temática en nuestro país, pero aún seguimos asumiendo datos de otras sociedades similares a la nuestra, es decir no estamos aportando con datos objetivos a través de la investigación empírica y cualitativa que nos permita mejorar la detección, la prevención y promoción así como la intervención. 


\section{REFERENCIAS BIBLIOGRAFICAS}

Batista Cardos o, M. (1994). «Mitos y Realidades sobre el maltrato y otros contra la mujer» Rev. de Psicología, Año $1 \mathrm{~N}^{\circ} 1$ Octubre Lima-Perú

Bronfenbrenner, U. (1987). La ecología del desarrollo humano. Paidos, Barcelona.

Castro, A. (1997). Prevalencia de la violencia en el Perú. PROMUDEH. Lima.

Castanedo, c.; Grupos de encuentro en terapia gestalt. Edil. Herder, México, 1990

Castanedo y otros; Seis enfoques psicoterapéuticos. Edil. Manual Moderno, México, 1993

CIPRES (1994). «Grupo de hombres contra la violencia» En la búsqueda de una nueva masculinidad». Puerto Rico.

CEDRO (1997). «Intervención sistémica en maltrato infantil y violencia intrafamiliar» C.E.F, Lima- Perú.

CENDOC- MUJER (1995). «Violencia Doméstica « Paquete Informativo Lima - Perú.

CORSI y COLBS. (1997). Violencia Familiar. Una mirada interdisciplinaria sobre un grave problema social. Paidos. Argentina.

DEMUS (1995). «Violencia en la relación de pareja: una búsqueda en la subjetividad de las mujeres». LimaPerú.

Fagan y Sheperd; Teoría y técnica de la terapia gestalt. Edil. Amorrurto Editores, México. 1980

Ginger, S. y Ginger, A. (1993). La Gestalt: una Terapia de Contacto. El Manual Moderno. Mexico

Jacobson, N.; Gottman, 1. (2001). Hombres que agraden a las mujeres. Como poner fin a las relaciones abusivas. Paidos Ibérica S.A. España.

Kahle, Anne-Barbel (2000). ¡Doma tu ira!. Apostrofe. España.

LoLi, Silvia (1990). «La violencia enmascarada». Edit. Viva $\mathrm{N}^{\circ} 17$, Lima.

Nieto Degregori, L (1996). « ¿ Callejón Oscuro?»Violencia contra la mujer en los barrios marginales del Cuzco. Cuzco-Perú

Nirvana González (1996). Violencia Doméstica: El ciclo perverso: «Derecho a vivir sin violencia».

Perls, Frederick (1990). El enfoque Guestáltico. Testimonios de Terapia. Cuatro Vientos. Santiago de Chile.

Perls, Frederick (1990). Dentro y fuera del tarro de basura. Autobiografía. Cuatro Vientos. Santiago de Chile

Pierre Pelletier (1997). «Violencia Conyugal». Flora Tristan. Lima- Perú.

Pimentel Sevilla, C (1998). «Familia, Violencia y Barriada «Tipacom. Lima Perú

Pimentel Sevilla, C (1995).«Violencia, Familia y Niñez» CECOSAM. Lima Perú.

PROMUDEH (2001). Programa Nacional contra la violencia familiar y sexual. Lima.

Rico Nieves (1996). «Violencia de Género un problema de derechos Humanos» CEPAL Perú.

Schnake, Adriana (1995) Los diálogos del cuerpo. Cuatro Vientos. Santiago de Chile

Tamayo, Luliana (1995).»La Violencia contra la Mujer en el Perú« Lima Perú

Ugarte, Dora (1989). «Violencia y Paz».

Centro de Psicoterapia Psicoanalítica Lima-Perú

Walker, Leonore (1994).» Curso de Asistencia psicológica a mujeres golpeadas». Argentina

Yontef, Gary (1997). Proceso y Diálogo en Psicoterapia Gestáltica. Cuatro Vientos.

Santiago de Chile. 